The Astrophysical Journal, 677:441-447, 2008 April 10

(C) 2008. The American Astronomical Society. All rights reserved. Printed in U.S.A.

\title{
GRB 070125: THE FIRST LONG-DURATION GAMMA-RAY BURST IN A HALO ENVIRONMENT ${ }^{1}$
}

\author{
S. Bradley Cenko, ${ }^{2}$ Derek B. Fox, ${ }^{3}$ Brian E. Penprase, ${ }^{4}$ Antonio Cucchiara, ${ }^{3}$ Paul A. Price, ${ }^{5}$ Edo Berger, ${ }^{6,7,8}$ \\ Shri R. Kulkarni, ${ }^{9}$ Fiona A. Harrison, ${ }^{2}$ Avishay Gal-Yam, ${ }^{10}$ Eran O. Ofek, ${ }^{9}$ Arne Rau, ${ }^{9}$ \\ Poonam Chandra, ${ }^{11,12}$ Dale A. Frail, ${ }^{13}$ Mansi M. Kasliwal, ${ }^{9}$ Brian P. Schmidt, ${ }^{14}$ \\ Alicia M. Soderberg, ${ }^{9}$ P. Brian Cameron, ${ }^{9}$ and Kathy C. Roth ${ }^{15}$ \\ Received 2007 May 24; accepted 2007 November 13
}

\begin{abstract}
We present the discovery and high signal-to-noise ratio spectroscopic observations of the optical afterglow of the long-duration gamma-ray burst GRB 070125 . Unlike all previously observed long-duration afterglows in the redshift range $0.5 \lesssim z \lesssim 2.0$, we find no strong (rest-frame equivalent width $W_{r} \gtrsim 1.0 \AA$ ) absorption features in the wavelength range 4000-10000 $\AA$. The sole significant feature is a weak doublet that we identify as $\mathrm{Mg}$ II $\lambda \lambda 2796\left(W_{r}=0.18 \pm\right.$ $0.02 \AA), 2803\left(W_{r}=0.08 \pm 0.01 \AA\right)$ at $z=1.5477 \pm 0.0001$. The low observed $\mathrm{Mg}$ II and inferred H I column densities are typically observed in galactic halos, far away from the bulk of massive star formation. Deep groundbased imaging reveals no host directly underneath the afterglow to a limit of $R>25.4$ mag. Either of the two nearest blue galaxies could host GRB 070125 ; the large offset $(d \geq 27 \mathrm{kpc})$ would naturally explain the low column densities. To remain consistent with the large local (i.e., parsec scale) circumburst density inferred from broadband afterglow observations, we speculate that GRB 070125 may have occurred far away from the disk of its host in a compact star-forming cluster. Such distant stellar clusters, typically formed by dynamical galaxy interactions, have been observed in the nearby universe and should be more prevalent at $z>1$, where galaxy mergers occur more frequently.
\end{abstract}

Subject headings: gamma rays: bursts

\section{INTRODUCTION}

The connection between long-duration ( $\Delta t \gtrsim 2 \mathrm{~s}$ ) gamma-ray bursts (GRBs) and hydrogen-stripped, core-collapse supernovae (i.e., Type $\mathrm{Ib} / \mathrm{c} \mathrm{SNe}$ ) is now well established in the nearby universe (see, e.g., Woosley \& Bloom 2006). At $z \gtrsim 0.3$, where the overwhelming majority of GRBs are detected (e.g., Berger et al. 2005a; Jakobsson et al. 2006b), Type Ib/c SNe are too faint, absorbed, and redshifted to be observed routinely with current facilities. Observations of the environments of distant GRBs,

\footnotetext{
1 Based in part on observations obtained at the Gemini Observatory, which is operated by the Association of Universities for Research in Astronomy, Inc., under a cooperative agreement with the NSF on behalf of the Gemini partnership: the National Science Foundation (US), the Particle Physics and Astronomy Research Council (UK), the National Research Council (Canada), CONICYT (Chile), the Australian Research Council (Australia), CNPq (Brazil), and CONICET (Argentina).

2 Space Radiation Laboratory, MS 220-47, California Institute of Technology, Pasadena, CA 91125; cenko@srl.caltech.edu.

3 Department of Astronomy and Astrophysics, Pennsylvania State University, 525 Davey Laboratory, University Park, PA 16802.

4 Pomona College Department of Physics and Astronomy, 610 North College Avenue, Claremont, CA 91711.

5 Institute for Astronomy, University of Hawaii, 2680 Woodlawn Drive, Honolulu, HI 96822.

${ }^{6}$ Observatories of the Carnegie Institute of Washington, 813 Santa Barbara Street, Pasadena, CA 91101.

7 Princeton University Observatory, Peyton Hall, Ivy Lane, Princeton, NJ 08544.

${ }^{8}$ Hubble Fellow.

9 Division of Physics, Mathematics, and Astronomy, 105-24, California Institute of Technology, Pasadena, CA 91125.

${ }_{10}$ Astrophysics Group, Faculty of Physics, Weizmaun Institute of Science, Rehovot 76100, Israel.

11 National Radio Astronomy Observatory, Charlottesville, VA, 22903.

12 Department of Astronomy, University of Virginia, P.O. Box 3818, Charlottesville, VA 22903.

13 National Radio Astronomy Observatory, Socorro, NM 87801.

14 Research School of Astronomy and Astrophysics, Australian National University, Mt. Stromlo Observatory, via Cotter Road, Weston Creek, ACT 2611, Australia.

${ }^{15}$ Gemini Observatory, 670 North A‘ohoku Place, Hilo, HI 96720.
}

however, are consistent with a massive star origin. GRB hosts are typically faint, blue, irregular galaxies with large specific star formation rates (star formation rate per unit stellar mass; Le Floc'h et al. 2003; Christensen et al. 2004). And within their hosts, GRB afterglows are found to be concentrated in the innermost regions, tracing the blue light from hot young stars even more strongly than Type Ib/c SNe (Bloom et al. 2002; Fruchter et al. 2006).

Bright GRB afterglows are therefore ideally suited to probe the dense gas in the very regions where stars are being formed. This stands in marked contrast to quasar (QSO) sight lines, which sample galaxies according to gas cross section and are therefore much more likely to probe the outer regions of galaxy halos (e.g., Prochaska et al. 2007a).

While the sample of afterglow absorption spectra suitable for elemental abundance studies is still quite small compared with QSOs, a general picture has nonetheless begun to take hold. GRB systems are characterized by (1) large metal equivalent widths and correspondingly large metal column densities (e.g., Metzger et al. 1997); (2) extremely high neutral hydrogen column densities (e.g., Hjorth et al. 2003), typically falling at $\log N(\mathrm{HI})>20.3$ (the socalled damped Ly $\alpha$ systems, or DLAs; Wolfe et al. 2005); and (3) subsolar metallicities, typically $Z \sim 0.1 Z_{\odot}$ (e.g., Berger et al. 2006). All three findings are consistent with a massive star origin for long-duration GRBs.

Here we present observations of a long-duration event, GRB 070125 , that does not fit neatly into this paradigm. Despite deep spectroscopy of a bright $(R \approx 19 \mathrm{mag})$ afterglow, we detect only weak $\mathrm{Mg}$ II absorption at $z=1.55$, a firm upper limit on the $\mathrm{Mg}$ II column density of the host galaxy. Coupled with the large offset between the afterglow and the nearest detected host galaxy candidate, our observations indicate that the large-scale (i.e., ISM) burst environment is dramatically different from all previously observed GRB hosts.

\section{OBSERVATIONS AND DATA REDUCTION}

GRB 070125 was discovered by the Inter-planetary Network at 07:20:45 UT on 2007 January 25 (Hurley et al. 2007). 


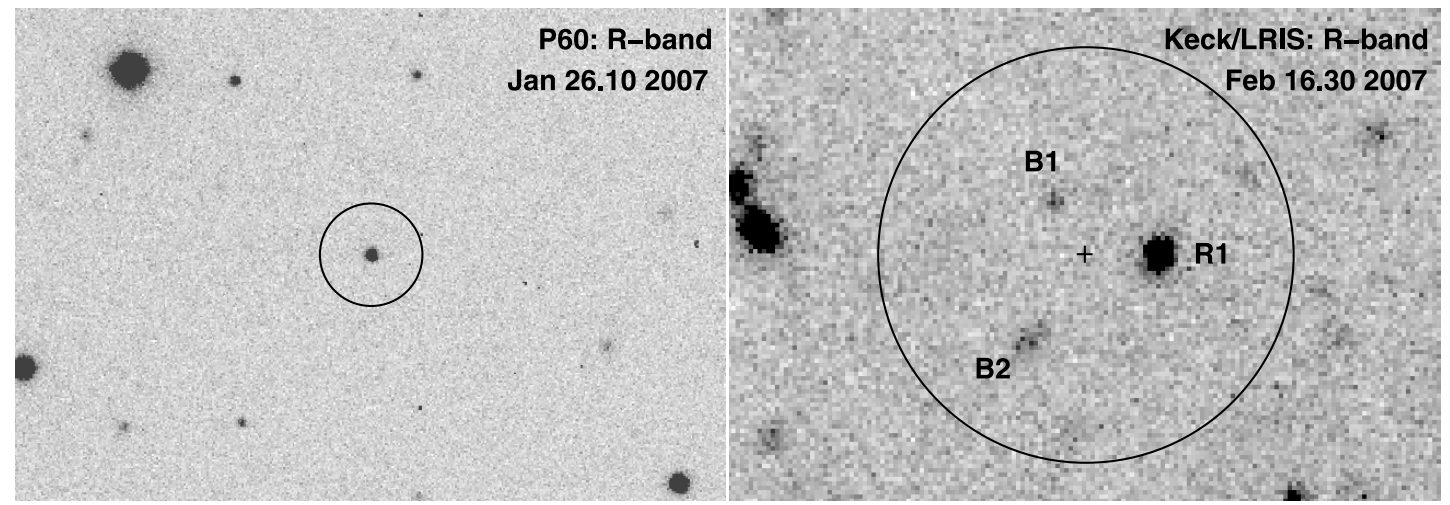

FIG. 1.-Optical imaging of the field of GRB 070125. Left: P60 R-band discovery image of the optical afterglow of GRB 070125 . The afterglow is centered inside a circle of radius $10^{\prime \prime}$. Right: Late-time Keck/LRIS $R$-band image of the field. The location of the afterglow is marked with a cross. The black circle again has a radius of $10^{\prime \prime}$. We find no evidence for an underlying host, to limits of $R>25.4 \mathrm{mag}, g^{\prime}>26.1 \mathrm{mag}$. The three galaxies nearest to the afterglow location are marked (B1, B2, and R1). R1, initially suggested as a possible host for GRB 070125 (Bloom et al. 2007), is a red, foreground galaxy $(z=0.897)$ with little current star formation. B1 and B2 are both quite blue $\left(g^{\prime}-R \approx 0\right)$, typical of long-duration GRB hosts. All images are oriented with north up and east to the left.

The burst was notable both for its brightness $\left(F_{\gamma}=1.75_{-0.15}^{+0.18} \times\right.$ $10^{-4} \mathrm{erg} \mathrm{cm}^{-2}$; Golenetskii et al. 2007) and its long duration ( $\Delta t \gtrsim 200 \mathrm{~s}$; Hurley et al. 2007; Golenetskii et al. 2007). The well-characterized prompt emission allowed measurements of the peak energy of the spectrum $\left(E_{\text {peak }}=367_{-51}^{+65} \mathrm{keV}\right.$; Golenetskii et al. 2007), as well as a "pseudoredshift" (Pélangeon 2006) of $z_{p}=1.3 \pm 0.3$ (Pelangeon \& Atteia 2007).

We began observing the field of GRB 070125 with the automated Palomar 60 inch $(1.5 \mathrm{~m})$ telescope (Cenko et al. 2006) at $02: 18: 59$ UT on 2007 January $26(\Delta t=19.0 \mathrm{hr})$. Inside the burst error circle, we found a bright, stationary source $(R=18.59 \pm$ $0.03 \mathrm{mag}$ ) not present in the Sloan Digital Sky Survey images of this field (Adelman-McCarthy et al. 2006) that we identified as the optical afterglow of GRB 070125 (Cenko \& Fox 2007; Fig. 1). Our subsequent broadband monitoring of the afterglow of GRB 070125 is presented in a separate work (Chandra et al. 2008).

We also undertook spectroscopic observations of GRB 070125 with the Gemini Multi-Object Spectrograph (GMOS; Hook et al. 2003) mounted on the $8 \mathrm{~m}$ Gemini North Telescope beginning on the night of 2007 January 26. For all spectra, we employed a $2 \times 2$ binning to increase the CCD signal-to-noise ratio $(\mathrm{S} / \mathrm{N})$, and we used the R400 grating and $1^{\prime \prime}$ slit. Our configuration resulted in a spectral resolution of $\sim 8 \AA$ and a dispersion of $1.34 \AA$ pixel $^{-1}$. The details of our observations are shown in Table 1.

All spectra were reduced in the IRAF ${ }^{16}$ environment using standard routines. Pairs of dithered spectra were subtracted to remove residual sky lines. Cosmic rays were removed using the LA Cosmic routine (van Dokkum 2001). Spectra were extracted optimally (Horne 1986), and wavelength calibration was performed first relative to CuAr lamps and then tweaked based on night sky lines in each individual image. In all cases, the resulting rms wavelength uncertainty was $\lesssim 0.3 \AA$. Both air-to-vacuum and heliocentric corrections were then applied to all spectra. Extracted spectra were divided through by a smoothed flux standard to remove narrowband ( $<50 \AA$ ) instrumental effects (Bessell 1999). Finally, telluric atmospheric absorption features were removed using the continuum from spectrophotometric standards (Wade \& Horne 1988; Matheson et al. 2000).

16 IRAF is distributed by the National Optical Astronomy Observatory, which is operated by the Association for Research in Astronomy, Inc., under cooperative agreement with the National Science Foundation.
Deep, late-time imaging to search for the host galaxy of GRB 070125 was taken with the Low-Resolution Imaging Spectrometer (LRIS: Oke et al. 1995) mounted on the $10 \mathrm{~m} \mathrm{Keck} \mathrm{I}$ telescope. LRIS employs a dichroic beam splitter, allowing simultaneous imaging in both $g^{\prime}$ - and $R$-band filters. We obtained $4 \times 300 \mathrm{~s}$ images at a mean epoch of 7:12:06.6 UT on 2007 February 16. Individual images were bias-subtracted and flatfielded using standard IRAF routines. Co-addition was performed using SWarp. ${ }^{17}$ The resulting $R$-band image is shown in Figure 1 (right panel).

\section{RESULTS}

In Figure 2 we show a sum of all four GMOS spectra obtained on the night of 2007 January $26(1 a-1 d$; see Table 1), normalized by the continuum. The strongest absorption feature present is a doublet at $\lambda \lambda 7124,7142 \AA$, with observed equivalent widths of $0.47 \pm 0.05 \AA\left(\lambda_{1}=7124.23 \pm 0.35 \AA\right)$ and $0.21 \pm 0.04 \AA$ $\left(\lambda_{2}=7142.45 \pm 0.42 \AA\right)$. Despite the weakness of the feature, the doublet is detected in separate co-additions of spectra from the two different instrumental configurations $\left(\lambda_{\text {central }}=6000 \mathrm{vs}\right.$. $8000 \AA$ ), providing strong confirmation of its reality (see Fig. 2 , right inset). Inspection of the two-dimensional spectra reveals that the only other significant absorption feature, at $\lambda=6283 \AA$, is offset slightly from the center of the trace. Furthermore, it is only detected in one instrumental configuration. We therefore believe that this feature is most likely an artifact of the data reduction process.

We find marginal ( $\leqslant 2 \sigma$ ) evidence for strengthening of both features in the doublet over the duration of our observations. Variability has been reported before in GRB afterglow spectra, both within the GRB host itself (time-dependent excitation caused by UV photons from the GRB afterglow; Vreeswijk et al. 2007) and in intervening absorbers (caused by variability in the GRB beam size relative to intervening clouds; Hao et al. 2007). However, because of the uncertainty of this result, we proceed using average values measured from the sum of all our spectra obtained on the night of 2007 January 26.

Based on the lack of Ly $\alpha$ absorption, we place an upper limit on the afterglow redshift of $z \leq 2.3$. Prochaska et al. (2007b) report a contemporaneous LRIS spectrum of the afterglow with coverage extending down to the atmospheric cutoff at $\lambda \approx 3000 \AA$. Based on the absence of damped Ly $\alpha$ absorption or Ly $\alpha$ forest

${ }^{17}$ See http://terapix.iap.fr. 
TABLE 1

Log of Spectroscopic Observations

\begin{tabular}{|c|c|c|c|c|c|c|}
\hline Identification & UT Date $^{\mathrm{a}}$ & $\begin{array}{l}\text { Age }^{\mathrm{b}} \\
\text { (days) }\end{array}$ & Primary Target & $\begin{array}{l}\text { Wavelength Coverage } \\
\text { (§) }\end{array}$ & Air Mass ${ }^{\mathrm{c}}$ & $\begin{array}{c}\text { Exposure Time } \\
\text { (s) }\end{array}$ \\
\hline $1 a \ldots$ & 2007 Jan 26.228 & 0.922 & Afterglow & $5900-10000$ & 1.98 & 1800 \\
\hline $1 b \ldots \ldots$ & 2007 Jan 26.250 & 0.944 & Afterglow & $5900-10000$ & 1.67 & 1800 \\
\hline $1 c \ldots \ldots \ldots \ldots \ldots \ldots \ldots \ldots \ldots \ldots \ldots \ldots$ & 2007 Jan 26.272 & 0.966 & Afterglow & $4000-8100$ & 1.45 & 1800 \\
\hline $1 d \ldots \ldots \ldots \ldots \ldots \ldots \ldots$ & 2007 Jan 26.294 & 0.989 & Afterglow & $4000-8100$ & 1.30 & 1800 \\
\hline $2 a \ldots \ldots \ldots \ldots \ldots \ldots \ldots \ldots \ldots \ldots \ldots$ & 2007 Jan 29.247 & 3.941 & Afterglow & $5900-10000$ & 1.60 & 1800 \\
\hline $2 b \ldots \ldots$ & 2007 Jan 29.269 & 3.963 & Afterglow & $5900-10000$ & 1.41 & 1800 \\
\hline $2 c$ & 2007 Jan 29.290 & 3.984 & Afterglow & $5900-10000$ & 1.27 & 1800 \\
\hline $2 d \ldots \ldots \ldots \ldots \ldots \ldots \ldots \ldots \ldots \ldots \ldots \ldots \ldots$ & 2007 Jan 29.312 & 4.006 & Afterglow & $5900-10000$ & 1.18 & 1800 \\
\hline 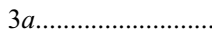 & 2007 Feb 4.340 & 9.992 & $R 1$ & $5900-10000$ & 1.06 & 2400 \\
\hline . & 2007 Feb 4.369 & 10.063 & $R 1$ & $5900-10000$ & 1.03 & 2400 \\
\hline
\end{tabular}

${ }^{\text {a }}$ UT at midpoint of exposure.

${ }^{b}$ Age in days from detection of the burst at 7:20:45 UT on 2007 January 25 (Hurley et al. 2007).

${ }^{c}$ Average air mass of exposure.

emission, they report a redshift upper limit of $z<1.4$. Given the weakness of the observed doublet, the expected weakness of the associated Ly $\alpha$ absorption (see below), and the decrease in sensitivity at the bluest LRIS wavelengths, we believe that this limit is too strict. Instead, we adopt a more conservative value of $z<1.8$ (corresponding to $\lambda_{\mathrm{Ly} \alpha} \leq 3500 \AA$ ) throughout this work.

Consistent with the above redshift constraint, we identify the observed doublet as $\mathrm{Mg}$ II $\lambda \lambda 2796,2803$ at $z=1.5477 \pm 0.0001$. Besides the observed wavelength ratio, we offer two additional pieces of evidence in support of this association. First, the observed equivalent width ratio $\left(\mathrm{Mg}_{\text {II }} 22796 / \mathrm{Mg}_{\text {II }} 22803\right)$ is consistent with the value of $2: 1$ predicted for weak, unsaturated absorption from this transition. Second, the $\mathrm{Mg}$ II $\lambda \lambda 2796,2803$ doublet is the strongest absorption feature observed in all GRB hosts identified in the redshift range $0.5 \lesssim z \lesssim 2.0$ (see below). Even if the system does not arise from the GRB host, this doublet is commonly found in intervening systems of both QSOs (Steidel \& Sargent 1992) and GRBs (Prochter et al. 2006). At this redshift, we place an upper limit on the rest-frame equivalent width of absorption from $\mathrm{Mg}_{\mathrm{I}} \lambda 2852$ of $W_{r}<0.06 \AA$ (Fig. 2, left inset).
Based on the observed $\mathrm{Mg}$ II equivalent widths, we can calculate corresponding column densities in the optically thin (i.e., unsaturated) limit:

$$
N=\frac{m_{e} c^{2}}{\pi e^{2}} \frac{W_{r}}{f \lambda^{2}}=1.13 \times 10^{20} \mathrm{~cm}^{-2} \frac{\left(W_{r} / \AA\right)}{(\lambda / \AA)^{2} f},
$$

where $f$ is the oscillator strength (from Morton 1991), $W_{r}$ is the rest-frame equivalent width, and $\lambda$ is the rest wavelength. The results are shown in Table 2. For the observed system we measure a column density of $\log N(\mathrm{Mg}$ II $)=12.61 \pm 0.05$. The corresponding upper limit on the $\mathrm{Mg}_{\mathrm{I}}$ column density is $\log N(\mathrm{Mg} \mathrm{I})<11.7$.

To compare our observed $\mathrm{Mg}$ II system with previous samples, we would like to know whether it arises from the GRB host or some intervening galaxy. To this end, we undertook a second epoch of GMOS spectroscopy on the night of 2007 January 29 ( $2 a-2 d$; see Table 1) to search for nebular emission lines at the location of the afterglow. While still bright enough to provide a reliable trace $(R \sim 21.5 \mathrm{mag})$ at this epoch, the decreased afterglow flux improved our sensitivity to faint emission lines. At $z=1.5477$, the only common line indicative of active star formation to fall in our bandpass is [O II 23727$]\left(\lambda_{\text {obs }} \approx 9497\right)$.

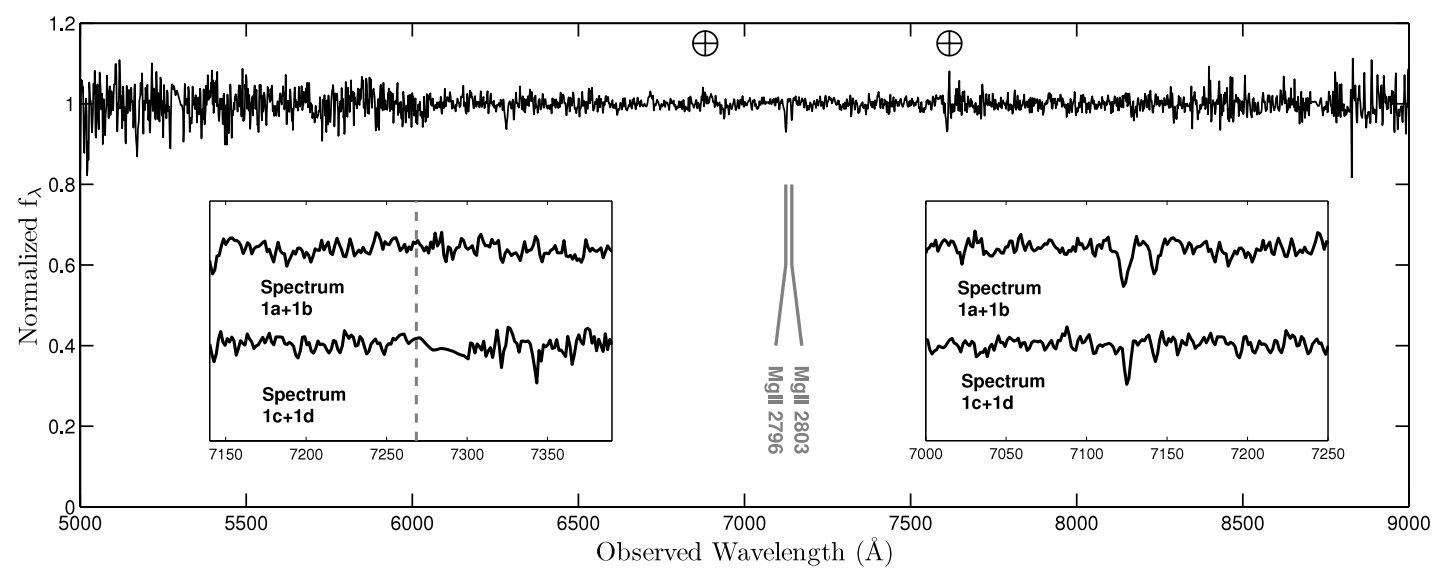

FIG. 2.- Gemini/GMOS spectrum of the afterglow of GRB 070125. In the primary plot we show the sum of all four spectra obtained the night of 2007 January 26 after

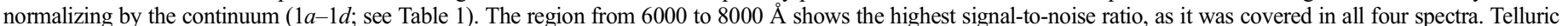

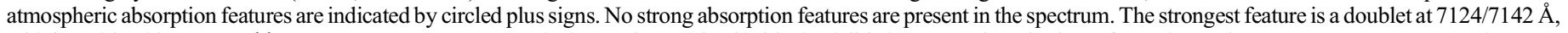

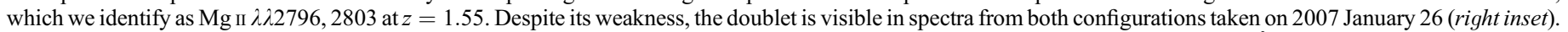
In the left inset, we zoom in on the $\mathrm{Mg}$ I $\lambda 2852$ transition at $z=1.55$. No absorption is detected in either configuration to $W_{r}<0.06 \AA$. 
TABLE 2

Absorption Line Identifications

\begin{tabular}{|c|c|c|c|c|c|c|}
\hline $\begin{array}{c}\text { Observed Wavelength } \\
(\AA)\end{array}$ & Identification & $\begin{array}{c}\text { Rest Wavelength } \\
(\AA)\end{array}$ & Redshift & $\begin{array}{c}\text { Rest Frame } \\
\text { Equivalent Width } \\
(\AA)\end{array}$ & Oscillator Strength $^{\mathrm{a}}$ & $\begin{array}{l}\text { Column Density } \\
\log \left(\mathrm{cm}^{-2}\right)\end{array}$ \\
\hline $7124.23 \pm 0.35 \ldots \ldots \ldots \ldots \ldots \ldots \ldots$ & $\mathrm{Mg}$ II & 2796.352 & $1.54769 \pm 0.00012$ & $0.18 \pm 0.02$ & 0.612 & $12.63 \pm 0.05$ \\
\hline $7142.45 \pm 0.42 \ldots \ldots \ldots \ldots \ldots \ldots \ldots$ & $\mathrm{Mg}$ II & 2803.531 & $1.54766 \pm 0.00015$ & $0.08 \pm 0.01$ & 0.305 & $12.58 \pm 0.06$ \\
\hline $7268.49^{\mathrm{b}} .$. & $\mathrm{Mg}_{\mathrm{I}}$ & 2852.964 & 1.5477 & $<0.06$ & 1.810 & $<11.7$ \\
\hline
\end{tabular}

a Reference: Morton (1991)

b The Mg I upper limits assume a redshift of $z=1.5477$ and a line width of $20 \mathrm{~km} \mathrm{~s}^{-1}$.

The presence of several bright night sky lines nearby significantly affected our sensitivity. Nonetheless, we put an upper limit on the observed flux ${ }^{18}$ from [O II 23727$]$ at $z=1.5477$ of $<5 \times$ $10^{-18} \mathrm{erg} \mathrm{cm}^{-2} \mathrm{~s}^{-1}$. Using the relation from Kennicutt (1998) this corresponds to an upper limit on the star formation rate of $<1 M_{\odot} \mathrm{yr}^{-1}$. This lies on the low end of star formation rates observed in previous GRB hosts (Christensen et al. 2004).

Without a secure emission-line redshift, we cannot determine the nature of the $\mathrm{Mg}$ II system (i.e., host or intervening). Nonetheless, because of our redshift constraints, $1.55 \leq z \leq 1.8$, the $\operatorname{Mg}$ II $\lambda \lambda 2796,2803$ transition from the host is guaranteed to fall

18 Throughout this work, we adopt a $\Lambda$ CDM cosmology with the latest parameters from $\operatorname{WMAP}\left(H_{0}=70.9 \mathrm{~km} \mathrm{~s}^{-1} \mathrm{Mpc}^{-1} ; \Omega_{m}=0.266 ; \Omega_{\Lambda}=1-\Omega_{m}\right.$; Spergel et al. 2007). within our observed bandpass (n.b. this is the case even if we apply our weaker $z \leq 2.3$ constraint). Therefore, even if the observed $\mathrm{Mg}$ II system is from an intervening galaxy, the measured equivalent widths are a firm upper limit on the presence of $\mathrm{Mg}$ II in the host system. In what follows we assume that $z=1.55$ is the redshift of the GRB host; all our conclusions below are only strengthened if $z>1.55$.

In Figure 3 we plot the observed $\mathrm{Mg}$ II $\lambda 2796$ rest-frame equivalent width for GRB 070125 compared to all previously observed GRB hosts. On the ordinate axis we plot the ratio between the 2796 and $2803 \AA$ components of the doublet. We find that GRB 070125 is an outlier on both axes. Clearly, the inferred column density is significantly lower than that of any other GRB host galaxy. Furthermore, the $\mathrm{Mg}_{\text {II }} \lambda 2796 / \mathrm{Mg}_{\text {II }} \lambda 2803$ ratio in all previous GRBs was $\approx 1$, indicating significant saturation. For saturated

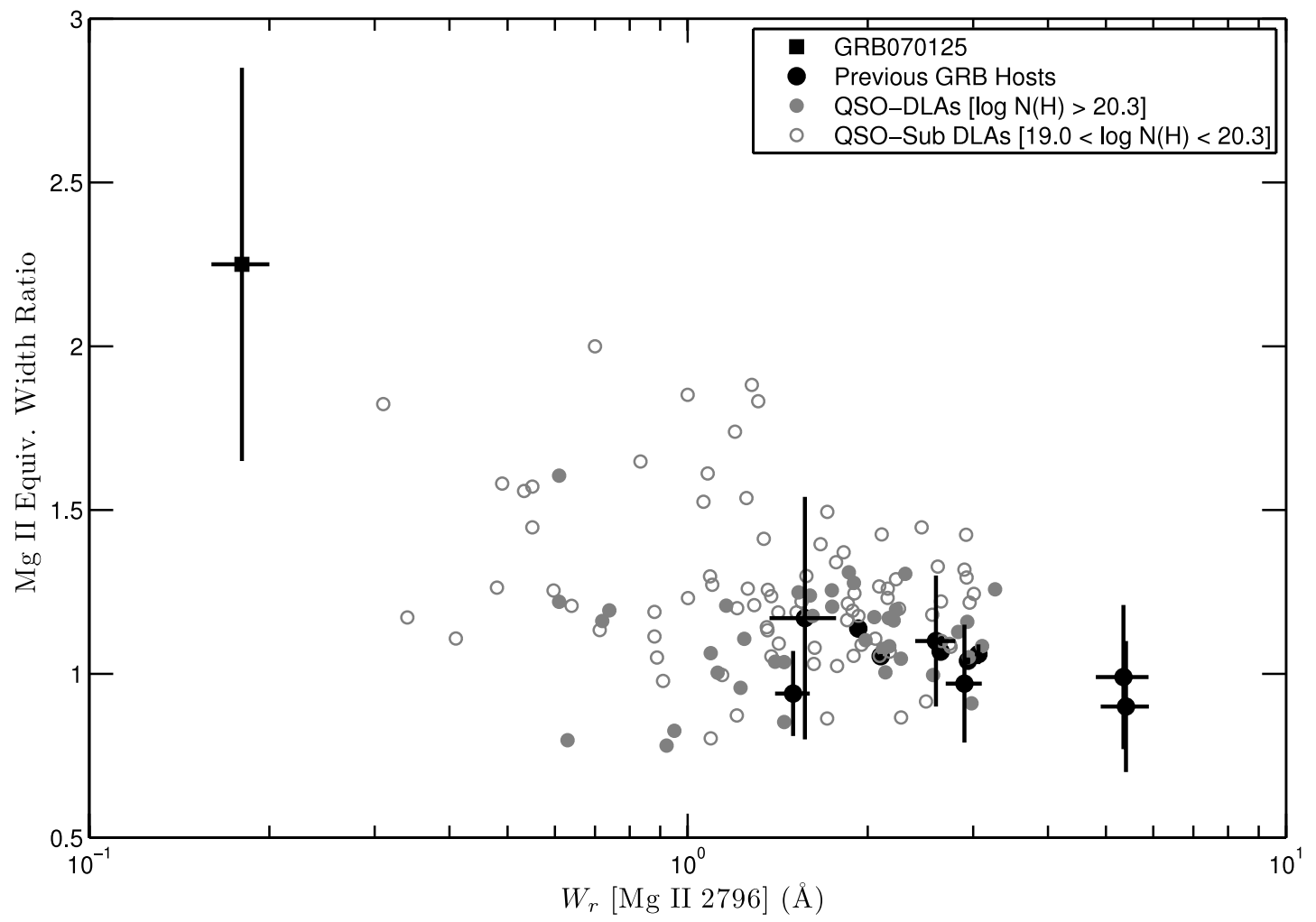

FIG. 3. - Mg II absorption in GRB host galaxies. Here we plot a compilation of all the equivalent width measurements of the $\mathrm{Mg}_{\mathrm{II}} \lambda 2796$ absorption feature in GRB host galaxies. On the $y$-axis, we plot the observed ratio between the $\mathrm{Mg}$ II $\lambda 2796$ and $\mathrm{Mg}$ II $\lambda 2803$ absorption lines. Ratios deviating from 2 indicate that the lines have become saturated and the corresponding optically thin column densities should be treated as lower limits. Thus, the factor of $\approx 10-15$ discrepancy between GRB 070125 and all previous GRB hosts actually underestimates the true difference in column densities. Shown in gray are analogous measurements for QSO-DLAs $[\log N(\mathrm{HI})>20.3 ;$ filled circles] and QSO-Sub DLAs [19.0 < $\log N(\mathrm{HI})<20.3$; open circles]. References: GRB 970508: Metzger et al. (1997); GRB 990123: Kulkarni et al. (1999); GRB 000926: Castro et al. (2003); GRB 010222: Mirabal et al. (2002); GRB 020813: Barth et al. (2003); GRB 030226: Shin et al. (2006); Klose et al. (2004); GRB 030328: Maiorano et al. (2006); GRB 041006: Soderberg et al. (2006b); GRB 051111: Penprase et al. (2006); Prochaska et al. (2007c); GRB 060418: Vreeswijk et al. (2007); Prochaska et al. (2007c); GRB 070208: Cucchiara et al. (2008, in preparation); QSOs: Rao et al. (2006). 
lines, the optically thin approximation (eq. [1]) breaks down and can significantly underestimate the true column density. The difference in $\mathrm{Mg}$ II column density between the host galaxy of GRB 070125 and all previous GRB hosts is therefore even larger than the factor of $\approx 10-15$ derived above.

To verify that the sample of GRB hosts used above is not biased toward strong $\mathrm{Mg}$ II absorbers, we searched through the GRB Circulars Network (GCN $)^{19}$ archive to review all reported longduration afterglow absorption spectra. Neglecting the most nearby events, $z \lesssim 0.3$, for which the strongest absorbers still lie in the $\mathrm{UV}$, we find only a single report of a high signal-to-noise ratio spectrum absent any absorption features in the optical bandpass (GRB 061021; Thoene et al. 2006). Alternatively, of the 17 longduration events with a reported redshift and spectral coverage of the host $\mathrm{Mg}$ II $\lambda \lambda 2796,2803$ transition, but without reported equivalent widths (i.e., not included in Fig. 3), 16 report a detection of this doublet. The sole exception, a spectrum of GRB 050802, contains several absorption features, but their identification was uncertain (Fynbo et al. 2005). We can therefore rule out a significant population of weak $\mathrm{Mg}$ II absorbers from GRB hosts at a high degree of confidence.

The detection of $\mathrm{Mg}$ I from GRB hosts is thought to indicate that these observations probe distances far away $(\geq 100 \mathrm{pc})$ from the GRB itself (Prochaska et al. 2007a; Vreeswijk et al. 2007). The first ionization energy of $\mathrm{Mg}$ is $7.6 \mathrm{eV}$, and therefore $\mathrm{UV}$ photons from the GRB afterglow are able to ionize any $\mathrm{Mg}$ in the circumburst medium to $\mathrm{Mg}$ II (subsequent ionizations beyond $\mathrm{Mg}$ II are likely shielded by neutral $\mathrm{H}$, as their ionization energies lie above 1 ryd). It is therefore important to determine whether we would expect to see $\mathrm{Mg}$ I absorption from GRB 070125, or whether the feature would be too weak to detect in our spectra.

Comparing the equivalent width ratio of $\mathrm{Mg}$ II $\lambda 2803$ (the weaker of the $\mathrm{Mg}$ II doublet, and therefore less saturated) to that of $\mathrm{Mg}$ I 22853 in previously observed GRB hosts, we find ratios ranging from 1.7 (GRB 970508; Metzger et al. 1997) to 2.8 (GRB 060418; Prochaska et al. 2007c; Vreeswijk et al. 2007). Again we note that these values are really a lower limit, as saturation will be more significant for the stronger $\mathrm{Mg}$ II $\lambda 2803$ feature. Nonetheless, we predict an $\mathrm{Mg}$ I $\lambda 2853$ rest-frame equivalent width of $\lesssim 0.03-0.05 \AA$. This is below our sensitivity limit, and we therefore believe that we would not be sensitive to $\mathrm{Mg}$ I absorption even if it were present at expected levels.

To convert our measured Mg column density (assumed to be dominated by $\mathrm{Mg}$ II) to neutral hydrogen, we must estimate the ratio of $\mathrm{Mg}: \mathrm{H}$ in the host. Previous GRB host metallicities fall in the range $-2.0 \lesssim[\mathrm{M} / \mathrm{H}] \lesssim-0.5$ (Berger et al. 2005b; Prochaska et al. 2007a), much like QSO-DLAs (Prochaska et al. 2003). At $z<2$, no QSO-DLA has ever been observed with $[\mathrm{M} / \mathrm{H}]<-2$ (Wolfe et al. 2005), and a near-solar metallicity is difficult to reconcile with a star-forming galaxy at $z=1.55$. If we neglect the cold, dense, disk depletion model, ruled out for all GRB hosts observed to date (Savaglio et al. 2003; Berger et al. 2005b; Penprase et al. 2006), all other environments predict an $\mathrm{Mg}$ depletion of $[\mathrm{Mg} / \mathrm{M}] \approx-0.5$ (Savage \& Sembach 1996). With the above limits, we estimate the neutral hydrogen column density to fall within $18.0 \lesssim \log N\left(\mathrm{H}_{\mathrm{I}}\right) \lesssim 19.5$ (using solar abundances from Asplund et al. 2005).

The implied $\mathrm{Mg}: \mathrm{H}$ ratio is in good agreement with previously observed GRBs, as well as the broader sample of QSOs. While $N(\mathrm{Mg}$ II $)$ and $N(\mathrm{H} \mathrm{I})$ have never been accurately measured simultaneously in a single GRB host, two events provide upper limits: $\left[\mathrm{Mg}_{\mathrm{II}} / \mathrm{H}_{\mathrm{I}}\right]>-2.7$ for GRB 000926 (Fynbo et al. 2002;

19 See http://gcn.gsfc.nasa.gov/gcn3_archive.html.
Castro et al. 2003), and $\left[\mathrm{Mg}_{\mathrm{II}} / \mathrm{H}_{\mathrm{I}}\right]>-1.6$ for GRB 030226 (Klose et al. 2004; Shin et al. 2006). For QSO-DLAs at $z<1.65$, the mean equivalent width for the $\mathrm{Mg}$ II $\lambda 2796$ transition is $1.8 \AA$, while for sub-DLAs $\left[19.0 \leq \log N\left(\mathrm{H}_{\mathrm{I}}\right) \leq 20.3\right]$ the corresponding value is $\left\langle W_{r}\right\rangle=1.6 \AA$ (Rao et al. 2006). For comparison, we also plot these QSO-Mg II systems in Figure 3. In a sample of eight weak $\left(W_{r}<0.3 \AA\right) \mathrm{Mg}$ II absorbers, Churchill et al. (1999) found that all had $\log N\left(\mathrm{H}_{\mathrm{I}}\right)<19.0$. In fact, six of the eight systems exhibited no sign of a Lyman limit break, indicating not only $\log N\left(\mathrm{HI}_{\mathrm{I}}\right)<17$, but also that neutral hydrogen was optically thin in those clouds (Tytler 1982).

An alternative way to determine the neutral hydrogen density is by modeling the afterglow spectral energy distribution (SED). Our models of the X-ray spectrum and optical SED do not require any dust extinction in addition to the Galactic component (Chandra et al. 2008; see also Racusin \& Vetere 2007). However, because of the relatively large Galactic column $[N(\mathrm{H}) \approx 5 \times$ $10^{20} \mathrm{~cm}^{-2}$; Dickey \& Lockman 1990] and the large effect of redshift on dust obscuration, these limits are not nearly as constraining as those derived from the optical spectrum.

All told, we have strong evidence that the neutral hydrogen column density in the host of GRB 070125 is quite low: $\log N\left(\mathrm{HI}_{\mathrm{I}}\right)<19.5$, if not significantly smaller. Such densities are usually associated with galaxy halos, and stand in contrast with the sample of previously observed GRB hosts, which are commonly attributed to a disk population. The sample of previously observed GRB hosts has a median $\log N(\mathrm{HI}) \approx 21.3$, with an observed standard deviation of 0.9 dex (Jakobsson et al. 2006a). In other words, to fall within $1 \sigma$ of the known distribution, a GRB host must be a DLA. Only three previous events, GRBs 021004 (Möller et al. 2002), 050908 (Foley et al. 2005), and 060607 (Jakobsson et al. 2006a), had measured $\log N(\mathrm{H} \mathrm{I})<$ 19.5 , and none report significantly lower values. All three exhibited strong absorption from other metals in addition to Ly $\alpha$, again distinguishing them from the mostly featureless spectrum of GRB 070125.

In our late-time imaging of the field of GRB 070125 (Fig. 1, right panel), we find no host directly underneath the afterglow location to limits of $R>25.4 \mathrm{mag}$ (Vega), $g^{\prime}>26.1 \mathrm{mag}(\mathrm{AB})$. Using a synthetic spectrum of a star-forming galaxy from Kinney et al. (1996) we estimate a limit on the absolute magnitude of $M_{V}>-19.2$ mag for any underlying host. Many GRB host galaxies are fainter than $M_{V}>-19.2$ (Fruchter et al. 2006), so it is entirely possible that our limits are too shallow to detect the underlying emission. Nonetheless, because of the low-density environment, we also consider the possibility that the afterglow lies significantly further away from its host than the typical GRB ( $\leq 10 \mathrm{kpc}$; Bloom et al. 2002; Fruchter et al. 2006).

We identify three candidate host galaxies within $10^{\prime \prime}$ of the afterglow location: R1, B1, and B2 (see Fig. 1, right panel). R1, 3.6" to the west of the afterglow, is a large red $\left(g^{\prime}-R \approx 2.4 \mathrm{mag}\right)$ galaxy identified by Bloom et al. (2007) as a possible host for GRB 070125. Based on a Gemini-GMOS spectrum obtained on the night of 2007 February 4 ( $3 a-3 b$; see Table 1$)$, we identify a strong continuum break at $\sim 7500 \AA$ as the rest-frame $4000 \AA$ break. $\mathrm{Ca} \mathrm{H}+\mathrm{K}$ and $\mathrm{G}-$ band absorption confirm that the galaxy lies in the foreground at $z=0.897 .{ }^{20}$ The other two objects, B1 at $3.2^{\prime \prime}$ distance and B2 at a distance of 5.5", are both blue $\left(g^{\prime}-\right.$ $R \approx 0 \mathrm{mag}$ ) and compact, more typical of long-duration GRB hosts (Le Floc'h et al. 2003). Unfortunately neither galaxy fell on the slit in any of our spectra.

\footnotetext{
20 At a distance of $3.6^{\prime \prime}, R 1$ would need to be extremely massive $\left(\sim 10^{13} M_{\odot}\right)$ for gravitational lensing to significantly affect the afterglow.
} 


\section{DISCUSSION}

Finally, we speculate on the origin of GRB 070125. At first glance, a compact binary progenitor system, as has been argued to explain most short-duration bursts (Eichler et al. 1989), seems appealing for GRB 070125: the large host offset and low-density environment could naturally be explained by the asymmetric "kick" imparted to such systems as the members become $\mathrm{SNe}$ (Fryer et al. 1999; Bloom et al. 1999). The accretion disk formed in such a merger is expected to last only a fraction of a second (Narayan et al. 2001), a serious discrepancy with the observed duration. However, the recent discovery of two nearby, long-duration GRBs lacking associated supernova emission (Gal-Yam et al. 2006; Fynbo et al. 2006; Della Valle et al. 2006; Ofek et al. 2007) leads us to at least consider an origin not associated with massive stars.

In a separate work, Chandra et al. 2008 study the broadband afterglow emission from GRB 070125. Two findings from this study cast doubt on a compact binary merger origin for this event. First, the total energy release from GRB 070125 , including the collimation correction, is extreme even for long bursts $\left(E \gtrsim 10^{52} \mathrm{erg}\right)$. Short-duration bursts typically are less energetic $\left(E \lesssim 10^{50} \mathrm{erg}\right.$; Fox et al. 2005), although the higher redshift examples discussed in Berger et al. (2007) appear to be more luminous, and may call this into question.

More importantly, however, based on the broadband SED (particularly the self-absorbed radio spectrum), we conclude that the local (parsec scale) circumburst density is quite high, even for typical long-duration afterglows $\left(n \sim 20 \mathrm{~cm}^{-3}\right.$ for a constantdensity environment). While this may seem inconsistent with the low Mg II column density derived from absorption spectroscopy, we instead consider the two observations the strongest evidence to date that afterglow studies and absorption spectroscopy probe distinct regions: the parsec-scale circumburst medium for the afterglow versus the more distant $(\geq 100 \mathrm{pc})$ ISM for absorption spectroscopy (Prochaska et al. 2007a; Vreeswijk et al. 2007). In the compact binary merger scenario, a large host offset should imply a low circumburst density $\left(n \lesssim 10^{-3} \mathrm{~cm}^{-3}\right)$, as has been seen for many short-duration bursts already (Fox et al. 2005; Soderberg et al. 2006a; Panaitescu 2006).

Because of the long duration, large local density, and large energy release, we return again to consider a massive star progenitor. Instead, we now must explain how a massive star could end up so far away from the dense disk of its host. For the closest putative host from our LRIS imaging, the observed offset of 3.2" corresponds to a projected distance of $\approx 27 \mathrm{kpc}$ at $z=1.5477$. To travel this distance in its short lifetime, a massive star would need an extremely large peculiar velocity: $\sim 10^{4} \mathrm{~km} \mathrm{~s}^{-1}$ for a 20 Myr lifetime. The fastest known object in the Galaxy is the Guitar pulsar, with a peculiar velocity of $1600 \mathrm{~km} \mathrm{~s}^{-1}$ (Cordes et al. 1993), while Galactic stars have been identified with peculiar velocities as large as $500 \mathrm{~km} \mathrm{~s}^{-1}$ (presumably accelerated by interacting with a black hole; Brown et al. 2006). It is much more probable that the progenitor was formed in situ.

Such a scenario has precedent in the local universe, where young, massive, compact star clusters have been found at large distances (i.e., several times the optical radius) either in extended UV disks (e.g., M83: Thilker et al. 2005; NGC 4625: Gil de Paz et al. 2005) or in tidal tails of interacting galaxies (e.g., "Antenna" system: Hibbard et al. 2005; Zhang et al. 2001; "Tadpole" galaxy: Jarrett et al. 2006). In some of the most strongly interacting systems, $\geq 10 \%$ of the current star formation is occurring in such clusters (Jarrett et al. 2006). Broadband surveys of nearby galaxies indicate that a significant fraction $(\leqslant 1 \%)$ of the current star formation in the local universe takes place in these extreme environments (D. Calzetti 2007, private communication). With our current understanding of hierarchical galaxy formation, such interactions should only increase in frequency as a function of look-back time. In retrospect, it is not entirely surprising that, of the $\sim 50$ long-duration GRBs with absorption spectra, we should discover such an event. While a thorough discussion of the relative frequency of such events is premature, the rarity of events like GRB 070125 implies that star formation in the outer regions of galaxies in the distant universe is likely not dramatically different from what we observe today.

We wish to thank R. Ellis for obtaining the Keck ToO imaging data. Some of the data were obtained with the Gemini Observatory under Program IDs GN-2006B-Q-21 and GN-2007A-Q-3. Some of the data presented herein were obtained at the W. M. Keck Observatory, which is operated as a scientific partnership among the California Institute of Technology, the University of California, and the National Aeronautics and Space Administration. The Observatory was made possible by the generous financial support of the W. M. Keck Foundation. S. B. C. and A. M. S. are supported by the NASA Graduate Student Research Program. E. B. is supported by NASA through Hubble Fellowship grant HST-HF-01171.01 awarded by STScI, which is operated by the Association of Universities for Research in Astronomy, Inc., for NASA, under contract NAS5-26555. A. G. acknowledges support by NASA through Hubble Fellowship grant HSTHF-01158.01 awarded by STScI. P. C. is supported by a Jansky fellowship. M. M. K. is supported by the Gordon and Betty Moore Foundation. GRB research at Caltech is supported by NASA and the NSF. The authors wish to recognize and acknowledge the very significant cultural role and reverence that the summit of Mauna Kea has always had within the indigenous Hawaiian community. We are most fortunate to have the opportunity to conduct observations from this mountain.

Facilities: Gemini:Gillett (GMOS), Keck:I (LRIS), PO:1.5m

\section{REFERENCES}

Adelman-McCarthy, J. K., et al. 2006, ApJS, 162, 38

Asplund, M., Grevesse, N., \& Sauval, A. J. 2005, in ASP Conf. Ser. 336, Cosmic Abundances as Records of Stellar Evolution and Nucleosynthesis, ed. T. G. Barnes III \& F. N. Bash (San Francisco: ASP), 25

Barth, A. J., et al. 2003, ApJ, 584, L47

Berger, E., Penprase, B. E., Cenko, S. B., Kulkarni, S. R., Fox, D. B., Steidel,

C. C., \& Reddy, N. A. 2006, ApJ, 642, 979

Berger, E., et al. 2005a, ApJ, 634, 501

2005b, Nature, 438, 988

2007, ApJ, 664, 1000

Bessell, M. S. 1999, PASP, 111, 1426

Bloom, J. S., Kulkarni, S. R., \& Djorgovski, S. G. 2002, AJ, 123, 1111

Bloom, J. S., Sigurdsson, S., \& Pols, O. R. 1999, MNRAS, 305, 763
Bloom, J. S., Starr, D., \& Blake, C. H. 2007, GCN Circ. 6054, http:// gcn.gsfc.nasa.gov/gen3/6054.gen3

Brown, W. R., Geller, M. J., Kenyon, S. J., \& Kurtz, M. J. 2006, ApJ, 647, 303 Castro, S., Galama, T. J., Harrison, F. A., Holtzman, J. A., Bloom, J. S., Djorgovski, S. G., \& Kulkarni, S. R. 2003, ApJ, 586, 128

Cenko, S. B., \& Fox, D. B. 2007, GCN Circ. 6028, http://gcn.gsfc.nasa.gov/ gcn $3 / 6028 . g c n 3$

Cenko, S. B., et al. 2006, PASP, 118, 1396

Chandra, P., et al. 2008, ApJ, submitted

Christensen, L., Hjorth, J., \& Gorosabel, J. 2004, A\&A, 425, 913

Churchill, C. W., Rigby, J. R., Charlton, J. C., \& Vogt, S. S. 1999, ApJS, 120,

51

Cordes, J. M., Romani, R. W., \& Lundgren, S. C. 1993, Nature, 362, 133 
Della Valle, M., et al. 2006, Nature, 444, 1050

Dickey, J. M., \& Lockman, F. J. 1990, ARA\&A, 28, 215

Eichler, D., Livio, M., Piran, T., \& Schramm, D. N. 1989, Nature, 340, 126

Foley, R. J., Chen, H.-W., Bloom, J. S., \& Prochaska, J. X. 2005, GCN Circ. 3949, http://gcn.gsfc.nasa.gov/gen3/3949.gen3

Fox, D. B., et al. 2005, Nature, 437, 845

Fruchter, A. S., et al. 2006, Nature, 441, 463

Fryer, C. L., Woosley, S. E., \& Hartmann, D. H. 1999, ApJ, 526, 152

Fynbo, J. P. U., et al. 2002, in Proc. MPA/ESO/MPE/USM Joint Astronomy Conf., Lighthouses of the Universe: The Most Luminous Celestial Objects and Their Use for Cosmology, ed. M. Gilfanov, R. Sunyaev, \& E. Churazov (Berlin: Springer), 187

2005, GCN Circ. 3749, http://gcn.gsfc.nasa.gov/gen3/3749.gcn3 . 2006, Nature, 444, 1047

Gal-Yam, A., et al. 2006, Nature, 444, 1053

Gil de Paz, A., et al. 2005, ApJ, 627, L29

Golenetskii, S., Aptekar, R., Mazets, E., Pal'Shin, V., Frederiks, D., \& Cline, T. 2007, GCN Circ. 6049, http://gcn.gsfc.nasa.gov/gen3/6049.gcn3

Hao, H., et al. 2007, ApJ, 659, L99

Hibbard, J. E., et al. 2005, ApJ, 619, L87

Hjorth, J., et al. 2003, ApJ, 597, 699

Hook, I., et al. 2003, Proc. SPIE, 4841, 1645

Horne, K. 1986, PASP, 98, 609

Hurley, K., et al. 2007, GCN Circ. 6024, http://gcn.gsfc.nasa.gov/gcn3/6024 gcn3

Jakobsson, P., et al. 2006a, A\&A, 460, L13 2006b, A\&A, 447, 897

Jarrett, T. H., et al. 2006, AJ, 131, 261

Kennicutt, R. C., Jr. 1998, ARA\&A, 36, 189

Kinney, A. L., Calzetti, D., Bohlin, R. C., McQuade, K., Storchi-Bergmann, T., \& Schmitt, H. R. 1996, ApJ, 467, 38

Klose, S., et al. 2004, AJ, 128, 1942

Kulkarni, S. R., et al. 1999, Nature, 398, 389

Le Floc'h, E., et al. 2003, A\&A, 400, 499

Maiorano, E., et al. 2006, A\&A, 455, 423

Matheson, T., Filippenko, A. V., Ho, L. C., Barth, A. J., \& Leonard, D. C. 2000, AJ, 120, 1499

Metzger, M. R., Djorgovski, S. G., Kulkarni, S. R., Steidel, C. C., Adelberger, K. L., Frail, D. A., Costa, E., \& Frontera, F. 1997, Nature, 387, 878

Mirabal, N., et al. 2002, ApJ, 578, 818
Möller, P., et al. 2002, A\&A, 396, L21

Morton, D. C. 1991, ApJS, 77, 119

Narayan, R., Piran, T., \& Kumar, P. 2001, ApJ, 557, 949

Ofek, E. O., et al. 2007, ApJ, 662, 1129

Oke, J. B., et al. 1995, PASP, 107, 375

Panaitescu, A. 2006, MNRAS, 367, L42

Pélangeon, A. 2006, in AIP Conf. Ser. 836, Gamma-Ray Bursts in the Swift Era, ed. S. S. Holt, N. Gehrels, \& J. A. Nousek (Melville: AIP), 149

Pelangeon, A., \& Atteia, J.-L. 2007, GCN Circ. 6059, http://gcn.gsfc.nasa.gov/ $\mathrm{gcn} 3 / 6059 . \mathrm{gcn} 3$

Penprase, B. E., et al. 2006, ApJ, 646, 358

Prochaska, J. X., Chen, H.-W., Dessauges-Zavadsky, M., \& Bloom, J. S. 2007a, ApJ, 666, 267

Prochaska, J. X., Gawiser, E., Wolfe, A. M., Castro, S., \& Djorgovski, S. G. 2003, ApJ, 595, L9

Prochaska, J. X., Roelofs, G., Bloom, J., \& Steeghs, D. 2007b, GCN Circ. 6032, http://gcn.gsfc.nasa.gov/gen3/6032.gen3

Prochaska, J. X., et al. 2007c, ApJS, 168, 231

Prochter, G. E., et al. 2006, ApJ, 648, L93

Racusin, J., \& Vetere, L. 2007, GCN Circ. 6030, http://gen.gsfc.nasa.gov/gen3/ 6030.gen 3

Rao, S. M., Turnshek, D. A., \& Nestor, D. B. 2006, ApJ, 636, 610

Savage, B. D., \& Sembach, K. R. 1996, ARA\&A, 34, 279

Savaglio, S., Fall, S. M., \& Fiore, F. 2003, ApJ, 585, 638

Shin, M.-S., et al. 2006, ApJ, submitted (astro-ph/0608327v1)

Soderberg, A. M., et al. 2006a, ApJ, 650, 261 . 2006b, ApJ, 636, 391

Spergel, D. N., et al. 2007, ApJS, 170, 377

Steidel, C. C., \& Sargent, W. L. W. 1992, ApJS, 80, 1

Thilker, D. A., et al. 2005, ApJ, 619, L79

Thoene, C. C., Fynbo, J. P. U., \& Jakobsson, P. 2006, GCN Circ. 5747, http:// gen.gsfc.nasa.gov/gen $3 / 5747 . g \mathrm{cn} 3$

Tytler, D. 1982, Nature, 298, 427

van Dokkum, P. G. 2001, PASP, 113, 1420

Vreeswijk, P. M., et al. 2007, A\&A, 468, 83

Wade, R. A., \& Horne, K. 1988, ApJ, 324, 411

Wolfe, A. M., Gawiser, E., \& Prochaska, J. X. 2005, ARA\&A, 43, 861

Woosley, S. E., \& Bloom, J. S. 2006, ARA\&A, 44, 507

Zhang, Q., Fall, S. M., \& Whitmore, B. C. 2001, ApJ, 561, 727 